\title{
Immune responses to SARS-CoV-2 in children of parents with symptomatic COVID-19
}

\section{Shidan Tosif ( $\nabla$ shidan.tosif@rch.org.au )}

Murdoch Children's Research Institute https://orcid.org/0000-0003-0022-1009

\section{Melanie Neeland}

Murdoch Children's Research Institute https://orcid.org/0000-0001-7301-9982

\section{Philip Sutton}

Murdoch Childrens Research Institute https://orcid.org/0000-0003-3129-1800

Paul Licciardi

Murdoch Childrens Research Institute https://orcid.org/0000-0001-6086-6285

\section{Sohinee Sarkar}

Murdoch Children's Research Institute https://orcid.org/0000-0001-9364-1920

\section{Kevin Selva}

University of Melbourne

\section{Lien Anh Ha Do}

Murdoch Childrens Research Institute

\section{Celeste Donato}

Murdoch Children's Research Institute

\section{Zheng Quan Toh}

Murdoch Children's Research Institute https://orcid.org/0000-0002-0282-5837

\section{Rachel Higgins}

Murdoch Children's Research Institute

\section{Carolien van de Sandt}

University of Melbourne https://orcid.org/0000-0002-4155-7433

\section{Melissa Lemke}

University of Michigan-Ann Arbor https://orcid.org/0000-0003-1860-4550

\section{Christina Lee}

University of Michigan-Ann Arbor

\section{Suzanne Shoffner}

University of Michigan-Ann Arbor

\section{Katie Flanagan}

Monash University https://orcid.org/0000-0002-1575-1953

\section{Kelly Arnold}

University of Michigan-Ann Arbor

Francesca Mordant 
University of Melbourne https://orcid.org/0000-0002-3754-2642

\section{Kim Mulholland}

Murdoch Childrens Research Institute

Julie Bines

Murdoch Children's Research Institute

Kate Dohle

Murdoch Children's Research Institute

\section{Dan Pellicci}

Murdoch Childrens Research Institute

\section{Nigel Curtis}

The University of Melbourne / The Royal Children's Hospital Melbourne / The Murdoch Children's Research Institute

\section{Sarah McNab}

Royal Children's Hospital

\section{Andrew Steer}

Murdoch Children's Research Institute

\section{Richard Saffery}

Murdoch Childrens Research Institute https://orcid.org/0000-0002-9510-4181

\section{Kanta Subbarao}

WHO Collaborating Centre for Reference and Research on Influenza https://orcid.org/0000-0003-17133056

\section{Amy Chung}

University of Melbourne https://orcid.org/0000-0003-0020-9704

\section{Katherine Kedzierska}

University of Melbourne https://orcid.org/0000-0001-6141-335X

\section{David Burgner}

Murdoch Children's Research Institute https://orcid.org/0000-0002-8304-4302

\section{Nigel Crawford}

Murdoch Children's Research Institute

\section{Article}

Keywords: Immunology, COVID-19, children, novel coronavirus, SARS-CoV-2

Posted Date: July 28th, 2020

DOI: https://doi.org/10.21203/rs.3.rs-47021/v1

License: (c) (i) This work is licensed under a Creative Commons Attribution 4.0 International License. Read Full License 
Version of Record: A version of this preprint was published at Nature Communications on November 11th, 2020. See the published version at https://doi.org/10.1038/s41467-020-19545-8. 


\section{Abstract}

Compared to adults, children with severe acute respiratory syndrome coronavirus 2 (SARS-CoV-2) have mild or asymptomatic infection, but the underlying immunological differences remain unclear. We describe clinical features, virology, longitudinal cellular and cytokine immune profile, SARS-CoV-2-specific serology and salivary antibody responses in a family of two parents with PCR-confirmed symptomatic SARS-CoV-2 infection and their three children, who were repeatedly SARS-CoV-2 PCR negative. Cellular immune profiles and cytokine responses of all children were similar to their parents at all timepoints. All family members had salivary anti-SARS-CoV-2 antibodies detected, predominantly IgA, that coincided with symptom resolution in 3 of 4 symptomatic members. Plasma from both parents and one child had IgG antibody detected against the S1 protein and virus neutralising activity ranging from just detectable to robust titers. Using a systems serology approach, we show that all family members demonstrated higher levels of SARS-CoV-2-specific antibody features than healthy controls. These data indicate that children can mount an immune response to SARS-CoV-2 without virological evidence of infection. This raises the possibility that despite chronic exposure, immunity in children prevents establishment of SARSCoV-2 infection. Relying on routine virological and serological testing may therefore not identify exposed children, with implications for epidemiological and clinical studies across the life-span.

\section{Main}

To date, children represent a small proportion of SARS-CoV-2 confirmed coronavirus disease (COVID-19) cases ${ }^{1-3}$. Children are predominantly infected from symptomatic household adult contacts ${ }^{4,5}$. Children have comparatively milder COVID-19 disease and up to one-third are asymptomatic ${ }^{6}$. The immunological basis for milder paediatric disease is unclear, but may be relevant to other viral pandemics where striking age-related epidemiological differences were observed ${ }^{7}$. In SARS-CoV-2 infection, reduced respiratory epithelial expression of the ACE2 receptor and trained innate immunity in children have been proposed 8,9 . Investigating immune responses to SARS-CoV-2 across all age groups is key to understanding disease susceptibility, severity determinants, and vaccine candidates. Detailed investigations of immune responses during SARS-CoV-2 infection have been reported in adults ${ }^{10-12}$, with exposure to SARS-CoV-2 causing specific $T$ cell responses without seroconversion ${ }^{13}$. Data on immune responses in children exposed to SARS-CoV-2 are limited.

Two parents (mother 38 years, and father 47 years) residing in Melbourne, Australia, attended a wedding inter-state without their children, in early March 2020. They returned home 3 days later and developed cough, coryza and subjective fevers, followed by lethargy and headache for a total of 14 (mother, A1) and 11 days (father, A2) (Fig. 1). Seven days after the onset of the parents' symptoms, child one (male 9 years, C1) developed mild cough, coryza, sore throat, abdominal pain and loose stools, and child 2 (male 7 years, C2) developed mild cough and coryza. The third child (female 5 years, C3) was asymptomatic. Eight days after the onset of the parents' symptoms, they were notified of an emerging outbreak of SARSCoV-2 traced to the wedding. The parents were SARS-CoV-2 PCR positive on nasopharyngeal (NP) swabs 
taken the same day. Repeated NP swabs from the children were negative for SARS-CoV-2. Physical distancing precautions were not feasible in the household. Child 3 had particularly close contact, sleeping in the parents' bed throughout the period both parents were unwell. All family members recovered fully without requiring medical care.

Serial samples, including blood, saliva, NP swabs, faeces and urine, were collected from all family members approximately every 2-3 days (Fig. 1). Nasopharyngeal swabs from the parents on days 8 and 12 were SARS-CoV-2 PCR positive. All NP, saliva and stool samples from the children were PCR negative for SARS-CoV-2. Nasopharyngeal swabs from the children were all positive for enterovirus by a multiplex respiratory viral panel on day 10.

We investigated the cellular immune response in peripheral blood mononuclear cells (PBMCs) from all family members on days 12,37 and 88 by flow cytometry. Both parents and children had high proportions of CD8 T cells at day 12 that subsequently decreased (Fig. 2A), a decline associated with a corresponding increase in the proportion of CD4 T cells in all samples. Strikingly low proportions of monocytes were observed on day 12 in all family members, particularly in C3 $(0.12 \%)$ relative to her siblings (average $0.5 \%$ ) and parents (average $0.88 \%$ ) (Fig. 2A). Monocytes returned to circulating proportions in all family members by day 37 (average $4.1 \%$ ) and day 88 (average $2.5 \%$ ). These signatures were also identified by unsupervised t-distributed stochastic neighbour embedding (tSNE) dimensionality reduction, where tSNE clusters corresponding to CD8 T, CD4 T and monocytes in parents and children showed identical sequential changes to those observed by manual gating (Fig. 2B). Low proportions of monocytes were observed in all circulating subsets with reductions in CD16 ${ }^{+}$subsets most evident (Fig. 2C). Both parents showed increases in central $\left(T_{C M}\right)$ and effector $\left(T_{E M}\right)$ memory CD8 $T$ cells by day 88 (Fig. 2D), and CD8 T cell expression of the exhaustion marker PD1 increased in all family members over time (Fig. 2E). CD4 $\mathrm{T}_{\mathrm{EM}}$ cells reduced over time in the parents, and one parent (A2) had a marked decline in the CD4 effector ( $\left.\mathrm{T}_{\text {EMRA }}\right)$ cell population (Fig. 2F).

The heterogeneous cellular immune responses observed in all family members at the first timepoint are consistent with emerging evidence on SARS-CoV-2 infection in adults ${ }^{14}$. In addition to CD8 T cell viral responses, depletion of innate immune cell subsets, including $\mathrm{CD} 16^{+}$monocytes, is an emerging, unique signature of COVID-19 ${ }^{15}$. We observed further alterations in the myeloid compartment in our whole blood analysis. Low proportions of neutrophils were evident in all family members at day 12 , particularly in C3 (5.1\%) relative to her siblings (average 10.4\%) and parents (average 15.5\%) (Fig. 2G). Circulating neutrophils returned to an average of $30.5 \%$ in children and $45.4 \%$ in parents by day 88 , a time point associated with the appearance of low-density immature neutrophils (SSChi $\left.C D 16^{+} \mathrm{CD} 14^{+/-}\right)$in PBMCs of all family members (Fig. 2B and $2 \mathrm{H}$ ). Pre- and immature- neutrophils in PBMC fractions have been recently described in SARS-CoV-2 infected adults ${ }^{16}$. In our study, parent A1 and all children had high proportions of eosinophils at all time points (Fig. 2G), in keeping with elevated eosinophils in SARS-CoV-2 infected patients during the recovery phase. Their role remains unclear ${ }^{17}$. 
Our analyses highlighted that active cellular immune responses in the family members were not accompanied by a corresponding increase in plasma cytokine levels, consistent with mild or absence of symptoms. We quantified 18 plasma cytokines using a custom multiplex bead array and only IL-8, MCP-1 and CCL5 (RANTES) were detectable (Fig. 2l), with levels remaining constant over time, excluding $\mathrm{C} 1$ and $\mathrm{C} 2$ who had a $\sim 2$-fold increase in RANTES levels at day 37 (Fig. 2I). A case of mild adult COVID-19 disease reported an identical plasma cytokine signature to that observed in our family members ${ }^{12}$.

To explore SARS-CoV-2 specific humoral immune responses, we first quantified salivary and plasma antibodies against the $S 1$ protein by ELISA. Saliva from all family members tested positive for IgA antibodies against the $\mathrm{S} 1$ protein at all timepoints (Fig. 3A). A2 had an increase in salivary anti-S1 IgA at day 12, one day after symptom resolution. $\mathrm{C} 1$ and $\mathrm{C} 2$ also had increased anti-S1 salivary IgA (Fig. 3A; day 25 and day 18 samples, respectively), coincident with symptom resolution. Anti-S1 IgM and IgG were present in most salivary samples, but with a less consistent pattern in family members. Both parents and C3 had detectable levels of plasma IgG and IgM to SARS-CoV-2 S1 protein at all timepoints (Fig. 3B). IgG levels increased between timepoints for parent A2; those for parent A1 remained stable. Levels of S1specific IgA in plasma were only detected in A1. Finally, A1 had a robust neutralising antibody response on days 12, 37 and 88 (titers 403, 226 and 160, respectively) (Fig. 3C). A2 and C3 had low level but detectable neutralizing antibody activity in sera on days 12 and 37 , respectively.

To further characterise whether the children had serological evidence of SARS-CoV-2 immunity despite being PCR negative, we undertook a systems serology analysis using a CoV-specific multiplex panel with the inclusion of additional aged-matched pre-pandemic healthy individuals. All family members, including the children, exhibited SARS-CoV-2-specific antibody features that differed from pre-pandemic controls (Fig. 4). This included serological signatures against the S1 protein, as well SARS-CoV-2 Trimer $S$, receptor binding domain (RBD) and S2. In addition, both parents, but not the children, had serological responses to other non-SARS-CoV-2 coronaviruses (Fig. 4C). Unsupervised hierarchical clustering analysis revealed that $\mathrm{C} 3$ clustered closest to her parents in all responses. C1 and C2, who had no evidence of a serologic response, clustered closest to the healthy controls whilst still exhibiting a SARSCOV-2 positive signature (Fig. 4C).

Our combined salivary and serological findings show that, despite having no virological evidence of infection, all three children developed antibody responses against various SARS-CoV-2 epitopes. Of the three children, C3, who remained asymptomatic throughout, demonstrated the most robust antibody response. We also observed that symptom resolution in A2, C1 and C2 coincided with a spike in salivary anti-S1 IgA, but not IgG. SARS-CoV-2 likely infects the salivary glands and is detectable in saliva ${ }^{18}$. Our observation therefore provides the first evidence that control of SARS-CoV-2 at the site of infection may be mediated by a mucosal IgA antibody response. This potential key role for mucosal antibodies in protection warrants confirmation in larger studies. Whilst enterovirus was identified in the children's respiratory panel, this is a common finding at our hospital and reflects recent exposure. The SARS-CoV-2 specific response identified in the saliva and serum would not be explained by this finding. 
This in-depth family case study provides novel insights into immunological responses in children exposed to SARS-CoV-2. Despite close contact with infected parents, PCR testing for SARS-CoV-2 was repeatedly negative in all children, who developed minimal or no symptoms. However, the children had similar cellular and SARS-CoV-2 specific antibody-mediated immune responses to their parents, suggesting that the children were infected with SARS-CoV-2 but, unlike the adults, mounted an immune response that was highly effective in restricting virus replication. Whether this family will be protected from reinfection with SARS-CoV-2 is uncertain, as only one parent demonstrated a robust neutralising antibody response. The discordance between the virological PCR results and clinical serological testing, despite an evident immune response, highlights limitations to the sensitivity of nasopharyngeal PCR and current diagnostic serology in children. Our findings emphasise the need for further detailed investigation of the immune response to SARS-CoV-2 to advance our understanding of exposure and protective immunity in children.

\section{Online Methods}

\section{SARS-CoV-2 detection}

RNA was manually extracted from $140 \mu \mathrm{L}$ of NP swabs and saliva, $280 \mu \mathrm{L}$ of urine and plasma and 140 $\mu \mathrm{L}$ of $20 \%\left(\mathrm{w} / \mathrm{v}\right.$ ) faecal suspension ${ }^{19}$ and then eluted in 50 to $60 \mu \mathrm{L}$ sterile, molecular water (Life technologies, Australia), using the QIAamp viral RNA kit (QIAgen GmbH, Hilden, Germany) according to the manufacturer's instructions. A previously published RT-PCR protocol targeting the RdRp gene was used on an $A B I 7500^{20}$. SARS-CoV-2 standard (Exact Diagnostic, US) was used as positive control for the PCR. Respiratory panel testing was by Ausdiagnostic viral panel.

\section{Plasma S1 protein ELISA}

The ELISA method used to measure IgG and IgM levels to SARS-COV-2 S1 protein was based on Amanat et al. ${ }^{21}$. Briefly, 96-well high-binding plates (Thermo Fisher Scientific) were coated with S1 (Sino Biological) diluted in PBS at $2 \mu \mathrm{g} / \mathrm{mL}$ and then incubated at $4{ }^{\circ} \mathrm{C}$ overnight. The following day, plates were washed with PBS containing $0.1 \%(\mathrm{v} / \mathrm{v})$ Tween 20 (PBS-T) and blocked with PBS containing $0.1 \%$ Tween and 10\% (w/v) skim milk (PBS-TSM) for 1 hour at room temperature (RT). Serial dilutions (3-fold) of plasma samples were prepared in PBS-TSM starting at 1:50. The blocking solution was removed and $100 \mu \mathrm{l}$ of each serial dilution was added to the plates for $2 \mathrm{~h}$ at RT. The plates were then washed three times with $200 \mu \mathrm{l}$ per well of PBS-T. Goat anti-human IgG- $(1: 10,000)$ or IgM- $(1: 5,000)$ horseradish peroxidase (HRP) conjugated secondary antibody (Southern Biotech) was prepared in PBS-TSM, and $50 \mu \mathrm{l}$ of this secondary antibody was added to each well for $1 \mathrm{~h}$. For IgA, $50 \mathrm{uL}$ of biotinylated IgA (1:5000) was diluted in PBS-T and added to each well for $1 \mathrm{~h}$, followed by the addition of StreptavidinHRP to each well for 30 min. Plates were washed with PBS-T followed by distilled water and 50 uL of 3.3', 5.5'-tetramethylbenzidine (TMB, Sera Care) substrate solution was added for $9 \mathrm{~min}$. The reaction was stopped by the addition of $50 \mathrm{uL}$ of $1 \mathrm{M}$ phosphoric acid and optical densities measured using a microplate reader (Bio-Tek) at $450 \mathrm{~nm}$ (630 nm reference filter). 


\section{Saliva S1 protein ELISA}

Saliva pooled under the tongue was drooled into a $50 \mathrm{~mL}$ tube and stored at $-80^{\circ} \mathrm{C}$ until analysed. Immuno MaxiSorp 96-well ELISA plates (Thermo Fisher Scientific) were coated overnight at $4{ }^{\circ} \mathrm{C}$ with $2 \mu \mathrm{g} / \mathrm{mL}$ recombinant SARS-CoV-2/2019-nCoV S1 protein (Sino Biologicals) diluted in PBS. Wells were blocked with $10 \%$ skim milk in PBST (PBS $+0.1 \%$ Tween 20 ) at room temperature for 1 hour. Two-fold serial dilutions of saliva samples in PBST were transferred to the ELISA plates (in duplicate) and incubated at room temperature for 1 hour. Saliva from an asymptomatic individual confirmed negative for SARS-CoV-2 by clinical testing was used as a negative control. Antibody binding was detected with biotinylated anti-human IgA (1:5000; Sigma-Aldrich) and IgG (1:10,000; Assay Matrix) for 1 hour at room temperature, then Streptavidin-HRP (1:5000; Life technologies) in PBST for $45 \mathrm{~min}$ at room temperature. Colour was developed with TMB solution (Sigma-Aldrich) and $\mathrm{H}_{2} \mathrm{O}_{2}$ with the reaction stopped using $2 \mathrm{M}$ $\mathrm{H}_{2} \mathrm{SO}_{4}$. Absorbance at $450 \mathrm{~nm}$ was read on a microplate reader.

\section{Microneutralisation assay}

SARS-CoV-2 isolate CoV/Australia/VIC01/2020 ${ }^{22}$ passaged in Vero cells was stored at $-80^{\circ} \mathrm{C}$.

Serial two-fold dilutions of heat-inactivated plasma were incubated with $100 \mathrm{TCID}_{50}$ of SARS-CoV-2 for 1 hour and residual virus infectivity was assessed in quadruplicate wells of Vero cells; viral cytopathic effect was read on day 5 . The neutralising antibody titre is calculated using the Reed/Muench method as previously described ${ }^{23,24}$.

\section{Systems serology}

Healthy participants

Age-matched children undergoing elective tonsillectomy (age 5-9) were recruited at the Launceston General Hospital (Tasmania) and, apart from fulfilling the criteria for tonsillectomy, they were considered otherwise healthy, showing no signs of immune compromise. Healthy adult donors (age 36-48) were recruited via the University of Melbourne. All healthy donors were recruited prior to SARS-CoV-2 pandemic. Heparinised blood was centrifuged for $10 \mathrm{~min}$ at $300 \mathrm{~g}$ to collect plasma, which was frozen at $-20^{\circ} \mathrm{C}$ until required.

Coupling of carboxylated beads

A custom CoV multiplex assay was designed and coupled as previous described ${ }^{25}$, with SARS-CoV-2 Spike 1 (Sino Biological), SARS-CoV-2 Spike 2, SARS-CoV Spike 1 (ACRO Biosystems, USA) and hCoV (229E, NL63, OC43) spikes (Sino Biologicals), as well as SARS-CoV-2 RBD (produced under HHSN272201400008C and obtained through BEI Resources, NIAID, NIH USA), SARS-CoV RBD (gift from Dale Godfrey) and both SARS-CoV-2 and HKU1 Trimeric Spikes (gift from Adam Wheatley). Tetanus toxoid (Sigma Aldrich) and influenza hemagglutinin (H1Cal2009; Sino Biological) were also added to the 
assay as positive controls. Antigens were covalently coupled to magnetic carboxylated beads (Bio Rad) using a two-step carbodiimide reaction and blocked with $0.1 \% \mathrm{BSA}$, before being resuspended and stored in PBS $0.05 \%$ sodium azide for use.

\section{Luminex bead-based multiplex assay}

The isotypes and subclasses of pathogen-specific antibodies present in collected plasma were assessed using the above multiplex assay as previously described ${ }^{25}$. Briefly, $20 \mu$ l of working bead mixture (1000 beads per bead region) and $20 \mu$ l of diluted plasma (final dilution 1:100) were added per well and incubated overnight at $4{ }^{\circ} \mathrm{C}$ on a shaker. Pathogen-specific antibodies were detected using 14 different detectors. One-step detection was done using phycoerythrin (PE)-conjugated mouse anti-human pan-IgG, IgG1-4, IgA1-2 (Southern Biotech; $1.3 \mu \mathrm{g} / \mathrm{ml}, 25 \mu \mathrm{l} /$ well), where detectors were added to the beads, washed then read by the MagPix. C1q protein (MP Biomedicals, USA) was first biotinylated (Thermo Fisher Scientific, USA), then tetramerized with Streptavidin R-PE (SAPE; Thermo Fisher Scientific) before dimers or tetrameric C1q-PE were being used in one-step detection. For the detection of FcyR-binding, twostep detection was done by first adding soluble recombinant FcyR dimers (higher affinity polymorphisms FcyRIla-H131, lower affinity polymorphisms FcyRlla-R131, FcyRllb, higher affinity polymorphisms FcyRllla-V158, lower affinity polymorphisms FcyRIlla-F158; $1.3 \mu \mathrm{g} / \mathrm{ml}, 25 \mu \mathrm{l} /$ well; gift from Bruce Wines and Mark Hogarth) to the beads, washing, followed by the addition of SAPE. Likewise for IgM, two-step detection was done using biotinylated mouse anti-human IgM (mAb MT22; MabTech; $1.3 \mu \mathrm{g} / \mathrm{ml}$, $25 \mu \mathrm{l} /$ well;), followed by SAPE. Assays were repeated in duplicate.

\section{Data Pre-processing for Systems Serology Analysis}

In the multivariate analysis, positive control antigens (Tetanus and H1Cal2009) were removed. All visit days were used for each individual. Data was right shifted and then $\log$ transformed $(\log 10(x+1))$. Right shifting was performed on each feature (detector-antigen pair) that contained negative values individually, by adding the minimum value for that feature to all samples within that feature. For all multivariate analysis the data was mean centered and variance scaled for each feature using the z-score function in Matlab.

Feature Selection

To determine the minimal set of features (signatures) needed to classify the various cohorts, a three-step process was used based on ${ }^{26}$. First, the data was randomly sampled without replacement to generate 2,000 subsets. All classes were resampled at the size of the smallest class for categorical outcomes, which corrected for any potential effects of class size imbalances during regularization. Elastic-Net regularization was then applied to each of the 2,000 resampled subsets to select features most associated with cohort classifications. The Elastic-Net hyperparameter, alpha, was set to have equal weights between the L1 norm and L2 norm associated with the penalty function for least absolute shrinkage and selection (LASSO) and ridge regression, respectively which allows for better analysis of collinear data, which may be eliminated in LASSO regression ${ }^{27}$. The frequency at which each feature 
was selected across the 2,000 iterations was used to determine the signatures by using a sequential stepforward algorithm that iteratively added a single feature into a PLSDA model starting with the feature that had the highest frequency of selection, to the lowest frequency of selection. Model prediction performance was assessed at each step and evaluated by 10 -fold cross-validation classification error. The model with the lowest classification error within a 0.01 difference between the minimum classification error was selected as the minimum signature. If only one feature was selected, the next best set of features was chosen. If consecutive feature sets were all equivalent, either the smallest or the largest set of features was chosen based on interpretability

\section{PLSDA}

Partial Least Squares Discriminant Analysis (PLSDA), performed in Eigenvectors PLS toolbox in Matlab, was used in conjunction with Elastic-Net, described above, to identify and visualize signatures that distinguish cohorts. This supervised method assigns a loading to each feature within a given signature, and identifies the linear combination of loadings (a latent variable) that best separates the categorical groups. A feature with a high loading magnitude indicates greater importance for separating the groups from one another. Each sample is then scored and plotted using their individual response measurements expressed through the latent variables (LVs). The scores and loadings can then be cross referenced to determine which features are loaded in association with which categorical groups (positively loaded features are higher in positively scoring groups etc). All models are created with 10 fold cross validation, where iteratively $10 \%$ of the data is left out as the test set, and the rest is used to train the model. Model performance is measured through calibration error (average error in the training set) as well as crossvalidation error (average error in the test set), with values near zero being best. All models were othronogonalized to enable clear visualization of results.

Hierarchical Clustering

Cohort classification clustering was visualized for the Healthy vs. Household Cohort and based on their feature selected signatures described above, using unsupervised average linkage hierarchical clustering of z-scored data. Euclidean distance was used as the distance metric.

\section{Software}

PLSDA models were completed using the Eigenvector PLS toolbox in Matlab. Hierarchical Clustering was completed using MATLAB 2017b (MathWorks, Natick, MA). PLSDA scores and loadings plots were plotted in Prism version 8.0.0.

\section{Flow cytometry of PBMC and whole blood}

Blood was collected in EDTA tubes from each participant at day 12, 37 and 88. Immediately following collection, $100 \mu \mathrm{l}$ of whole blood was aliquoted for flow cytometry analysis. The remaining EDTA blood samples were processed into plasma and PBMC as previously described ${ }^{28}$. For flow cytometry analysis of whole blood samples, whole blood was lysed with $1 \mathrm{~mL}$ of red cell lysis buffer for 10 minutes at room 
temperature. Cells were washed with $1 \mathrm{~mL}$ PBS and centrifuged at $350 \mathrm{xg}$ for 5 minutes. Following two more washes, cells were resuspended in PBS for viability staining using near infra-red viability dye according to manufacturers instructions. For flow cytometry analysis of freshly isolated PBMC, cells were washed in $1 \mathrm{~mL}$ PBS prior to viability staining using BV510 viability dye according to manufacturers instructions. For both whole blood and PBMC samples, the viability dye reaction was stopped by the addition of FACS buffer ( $2 \%$ heat-inactivated FCS in $2 \mathrm{mM}$ EDTA) and cells were centrifuged at $350 \mathrm{xg}$ for 5 minutes. Cells were then resuspended in human FC-block according to manufacturers instructions for 5 minutes at room temperature. The whole blood or PBMC antibody cocktails (Extended data Table 1) made up at $2 X$ concentration were added 1:1 with the cells and incubated for 30 minutes on ice. Following staining, cells were washed with $2 \mathrm{~mL}$ FACS buffer and centrifuged at $350 \mathrm{xg}$ for 5 minutes. Cells were then resuspended in $2 \%$ PFA for a 20 minute fixation on ice, washed, and resuspended in $150 \mu \mathrm{l}$ FACS buffer for acquisition using the BD LSR X-20 Fortessa. For all flow cytometry experiments, compensation was performed at the time of sample acquisition using compensation beads. Extended data Fig. 1 depicts the manual gating strategy for PBMC and whole blood samples.

Results were analysed (manual gating and tSNE analysis) using FlowJo Version 10.6 software. The tSNE plots was generated from a concatenated file containing 300,000 events $(20,000$ randomly selected live single cells per patient per time point). Manually gated results are presented as proportion of live cells or as proportion of parent gate (for PBMC) or as proportion of leukocyes (for whole blood). Data was plotted in Prism version 8.0.0.

\section{Plasma cytokines}

Plasma was diluted 1:2 and 1:4 for assessment of cytokines using the human soluble protein cytometric bead array flex sets (BD Biosciences) according to manufacturer's instructions. Cytometric bead array data were acquired on a BD LSR II X-20 Fortessa and analysed using the FCAP Array Software. The following 18 cytokines were quantified: IL-1a, IL-1 $\beta$, IL-6, IFNa, TNFa, MIP-1a, MCP-1, IL-8, RANTES, IL12p70, IL-10, IL-2, IL-5, IL-5, IL-9, IL-13, IFNy and IL-17A. All cytokines except for IL-8, MCP-1 and RANTES fell below the limit of detection of the assay at both dilutions and were excluded from future analysis. Results are reported in $\mathrm{pg} / \mathrm{mL}$ and plotted using Prism version 8.0.0.

\section{Declarations}

\section{Ethics}

Human experimental work was conducted according to the Declaration of Helsinki principles and according to the Australian National Health and Medical Research Council Code of Practice. All donors or their legal guardians provided written informed consent. The study was approved by the Human Research Ethics Committee (HREC) of the University of Melbourne (Ethics ID \#1443389.4, \#2056761, \#1647326, \#2056689, \#1955465) for healthy adults, Tasmanian Health and Medical HREC (H0017479) for healthy child donors. For the family case study, this project received ethical approval from The Royal Children's Hospital Melbourne Human Research Ethics Committee (HREC): HREC/63666/RCHM-2019. 


\section{ACKNOWLEDGEMENTS}

Dale Godfrey

Bruce Wines

P. Mark Hogarth

Adam Wheatley

Samantha Bannister

\section{FUNDING}

MRN is supported by a Melbourne Children's LifeCourse Fellowship, PVL is supported by NHMRC Career Development Fellowhsip (\#1146198), PS is supported by a DHB Foundation Fellowship. This work was supported by Jack Ma Foundation to KK, AWC, the Clifford Craig Foundation to KLF and KK, NHMRC Leadership Investigator Grant to KK (1173871), NHMRC Program Grant to KK (1071916), Research Grants Council of the Hong Kong Special Administrative Region, China (\#T11-712/19-N) to KK. AWC is supported by a NHMRC Career Development Fellowship (\#1140509), KK by NHMRC Senior Research Fellowship (1102792), CES has received funding from the European Union's Horizon 2020 research and innovation program under the Marie Skłodowska-Curie grant agreement (\#792532). This work is supported by Victorian Government's Medical Research Operational Infrastructure Support Program.

\section{References}

1. The epidemiological characteristics of an outbreak of 2019 novel coronavirus diseases (COVID-19) in China. Zhonghua Liu Xing Bing Xue Za Zhi 41, 145-151, doi:10.3760/cma.j.issn.02546450.2020.02.003 (2020).

2. Livingston, E. \& Bucher, K. Coronavirus disease 2019 (COVID-19) in Italy. JAMA 323, 1335-1335 (2020).

3. Severe Outcomes Among Patients with Coronavirus Disease 2019 (COVID-19) - United States, February 12-March 16, 2020. MMWR Morb Mortal Wkly Rep 69, 343-346, doi:10.15585/mmwr.mm6912e2 (2020).

4. Zhu, Y. et al. Children are unlikely to have been the primary source of household SARS-CoV-2 infections. medRxiv, doi:doi:2020.2003.2026.20044826. (2020).

5. 10.1542/peds.2020-1576

Posfay-Barbe, K. M. et al. COVID-19 in Children and the Dynamics of Infection in Families. Pediatrics, e20201576, doi:10.1542/peds.2020-1576 (2020).

6. Qiu, H. et al. Clinical and epidemiological features of 36 children with coronavirus disease 2019 (COVID-19) in Zhejiang, China: an observational cohort study. The Lancet Infectious Diseases, doi:10.1016/s1473-3099(20)30198-5 (2020). 
7. Short, K. R., Kedzierska, K. \& van de Sandt, C. E. Back to the Future: Lessons Learned From the 1918 Influenza Pandemic. Frontiers in cellular and infection microbiology 8, 343, doi:10.3389/fcimb.2018.00343 (2018).

8. Bunyavanich, S., Do, A. \& Vicencio, A. Nasal Gene Expression of Angiotensin-Converting Enzyme 2 in Children and Adults. JAMA, doi:10.1001/jama.2020.8707 (2020).

9. Zimmermann, P. \& Curtis, N. COVID-19 in Children, Pregnancy and Neonates: A Review of Epidemiologic and Clinical Features. The Pediatric infectious disease journal 39, 469-477, doi:10.1097/INF.0000000000002700 (2020).

10. Giamarellos-Bourboulis, E. J. et al. Complex Immune Dysregulation in COVID-19 Patients with Severe Respiratory Failure. Cell Host Microbe, doi:10.1016/j.chom.2020.04.009 (2020).

11. Wen, W. et al. Immune cell profiling of COVID-19 patients in the recovery stage by single-cell sequencing. Cell discovery 6, 31, doi:10.1038/s41421-020-0168-9 (2020).

12. Thevarajan, I. et al. Breadth of concomitant immune responses prior to patient recovery: a case report of non-severe COVID-19. Nature Medicine 26, 453-455, doi:10.1038/s41591-020-0819-2 (2020).

13. $10.1101 / 2020.06 .21 .20132449$

Gallais, F. et al. Intrafamilial Exposure to SARS-CoV-2 Induces Cellular Immune Response without Seroconversion. medRxiv, 2020.2006.2021.20132449, doi:10.1101/2020.06.21.20132449 (2020).

14. $10.1101 / 2020.05 .20 .106401$

Mathew, D. et al. Deep immune profiling of COVID-19 patients reveals patient heterogeneity and distinct immunotypes with implications for therapeutic interventions. bioRxiv, 2020.2005.2020.106401, doi:10.1101/2020.05.20.106401 (2020).

15. Wilk, A. J. et al. A single-cell atlas of the peripheral immune response in patients with severe COVID19. Nat Med, doi:10.1038/s41591-020-0944-y (2020).

16. $10.1101 / 2020.06 .03 .20119818$

Schulte-Schrepping, J. et al. Suppressive myeloid cells are a hallmark of severe COVID-19. medRxiv: the preprint server for health sciences, 2020.2006.2003.20119818, doi:10.1101/2020.06.03.20119818 (2020).

17. $10.1101 / 2020.06 .03 .20121582$

Rodriguez, L. et al. Systems-level immunomonitoring from acute to recovery phase of severe COVID19. medRxiv: the preprint server for health sciences, 2020.2006.2003.20121582, doi:10.1101/2020.06.03.20121582 (2020).

18. Xu, R. et al. Saliva: potential diagnostic value and transmission of 2019-nCoV. International Journal of Oral Science 12, doi:10.1038/s41368-020-0080-z (2020).

19. Cowley, D., Donato, C. M., Roczo-Farkas, S. \& Kirkwood, C. D. Novel G10P[14] rotavirus strain, northern territory, Australia. Emerging infectious diseases 19, 1324-1327, doi:10.3201/eid.1908.121653 (2013). 
20. Chan, J. F. et al. Improved Molecular Diagnosis of COVID-19 by the Novel, Highly Sensitive and Specific COVID-19-RdRp/Hel Real-Time Reverse Transcription-PCR Assay Validated In Vitro and with Clinical Specimens. Journal of clinical microbiology 58, doi:10.1128/JCM.00310-20 (2020).

21. Amanat, F. et al. A serological assay to detect SARS-CoV-2 seroconversion in humans. medRxiv: the preprint server for health sciences, doi:10.1101/2020.03.17.20037713 (2020).

22. Caly, L. et al. Isolation and rapid sharing of the 2019 novel coronavirus (SARS-CoV-2) from the first patient diagnosed with COVID-19 in Australia. The Medical journal of Australia 212, 459-462, doi:10.5694/mja2.50569 (2020).

23. Houser, K. V. et al. Prophylaxis With a Middle East Respiratory Syndrome Coronavirus (MERS-CoV)Specific Human Monoclonal Antibody Protects Rabbits From MERS-CoV Infection. J Infect Dis 213, 1557-1561, doi:10.1093/infdis/jiw080 (2016).

24. Subbarao, K. et al. Prior infection and passive transfer of neutralizing antibody prevent replication of severe acute respiratory syndrome coronavirus in the respiratory tract of mice. J Virol 78, 35723577, doi:10.1128/jvi.78.7.3572-3577.2004 (2004).

25. $10.1101 / 2020.05 .11 .20098459$

Selva, K. J. et al. Distinct systems serology features in children, elderly and COVID patients. medRxiv: the preprint server for health sciences, 2020.2005.2011.20098459, doi:10.1101/2020.05.11.20098459 (2020).

26. 10.1016/j.chom.2018.07.009

Gunn, B. M. et al. A Role for Fc Function in Therapeutic Monoclonal Antibody-Mediated Protection against Ebola Virus. Cell Host Microbe 24, 221-233 e225, doi:10.1016/j.chom.2018.07.009 (2018).

27. Hui Zou, T. H. Regularization and variable selection via the elastic net. J R Stat Soc B 67, 301-320 (2005).

28. Neeland, M. R. et al. Mass cytometry reveals cellular fingerprint associated with $\lg E+$ peanut tolerance and allergy in early life. Nat Commun 11, 1091, doi:10.1038/s41467-020-14919-4 (2020).

\section{Figures}

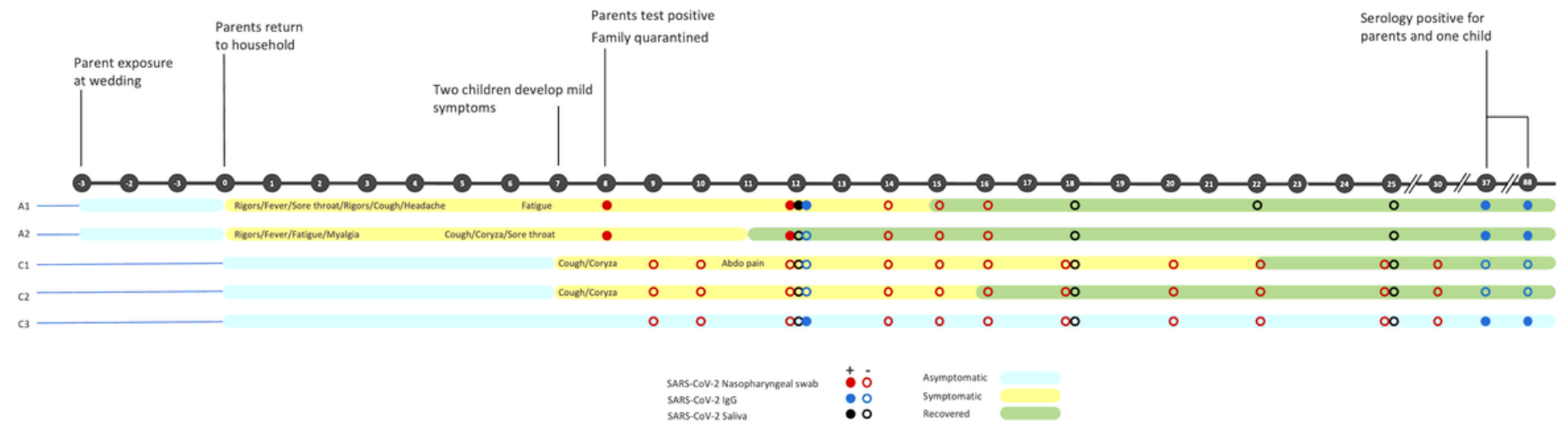

Figure 1 
Timeline of travel, exposure, symptoms, and selected results
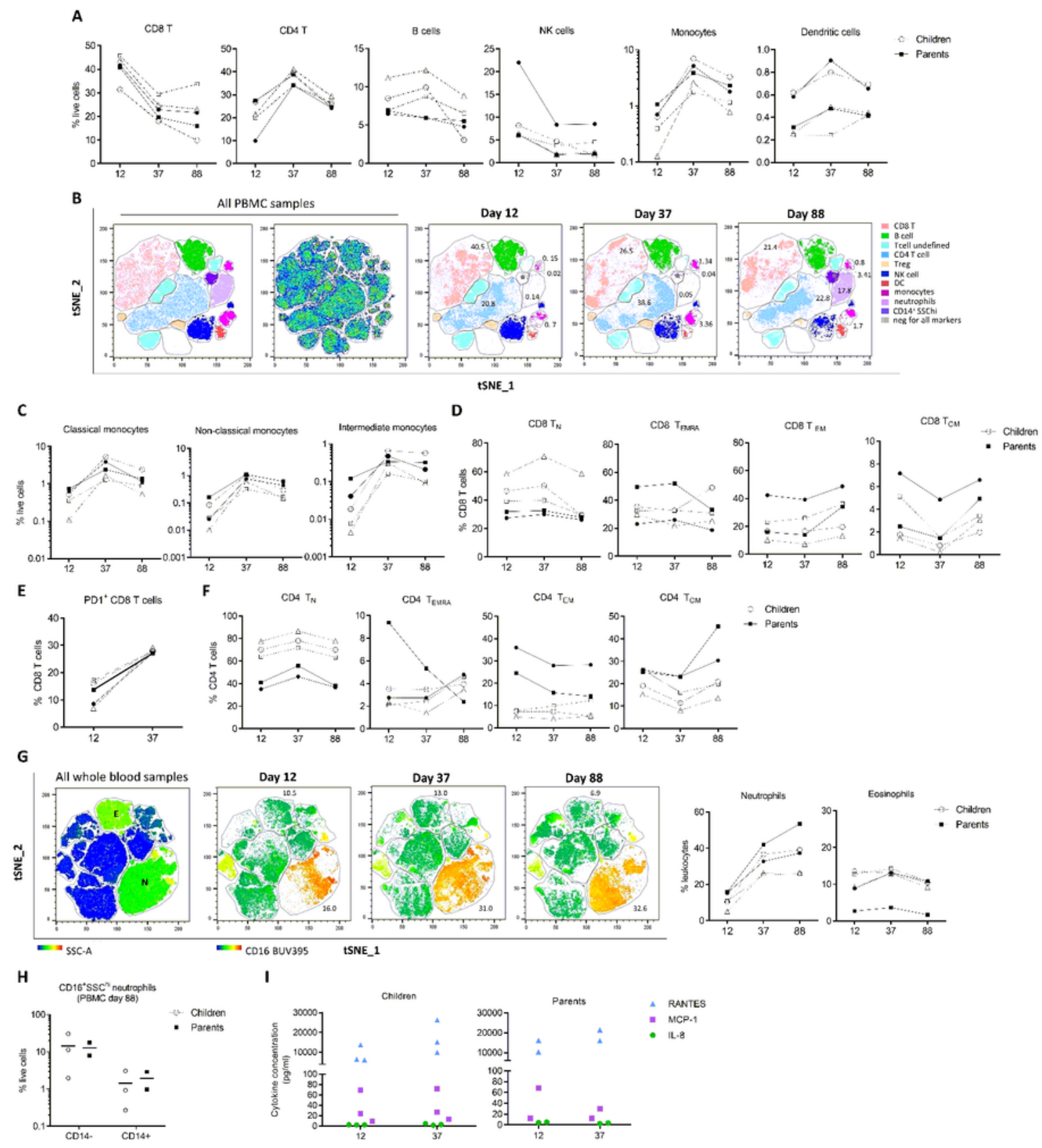

\section{Figure 2}

Longitudinal cellular immune profiling in parents and children. A. Major immune cell populations in PBMC at day 12, 37 and 88 in parents (solid line) and children (broken line) (A1 (closed circles), A2 (closed squares), C1 (open circles), C2 (open squares), C3 (open triangles)). B. tSNE dimensionality reduction of immune cell populations in all PBMC samples across the three time points. The tSNE plot 
was generated from a concatenated file containing 300,000 events $(20,000$ randomly selected live single cells per patient per time point). C. Frequency of monocyte subpopulations in PBMC from parents and children. D. Frequency of CD8 T cell naïve, effector and memory subpopulations in PBMC. E. Frequency of PD1 expressing CD8 T cells over time. F. Frequency of CD4 T cell naïve, effector and memory subpopulations in PBMC. G. TSNE dimensionality reduction of whole blood samples. The tSNE plot was generated from a concatenated file containing 300,000 events (20,000 randomly selected live single cells per patient per time point). Colouring depicts SSC and CD16 expression in tSNE islands. Granulocyte populations (neutrophils and eosinophils) are expressed as proportion of leukocytes. H. Frequency of low density CD16+SSChi neutrophils (CD14+ and CD14-) in PBMC fraction at day 88. I. Plasma cytokine concentration of three detectable cytokines, RANTES (blue), MCP-1 (purple) and IL-8 (green) in children and parents at day 12 and 37. 
A
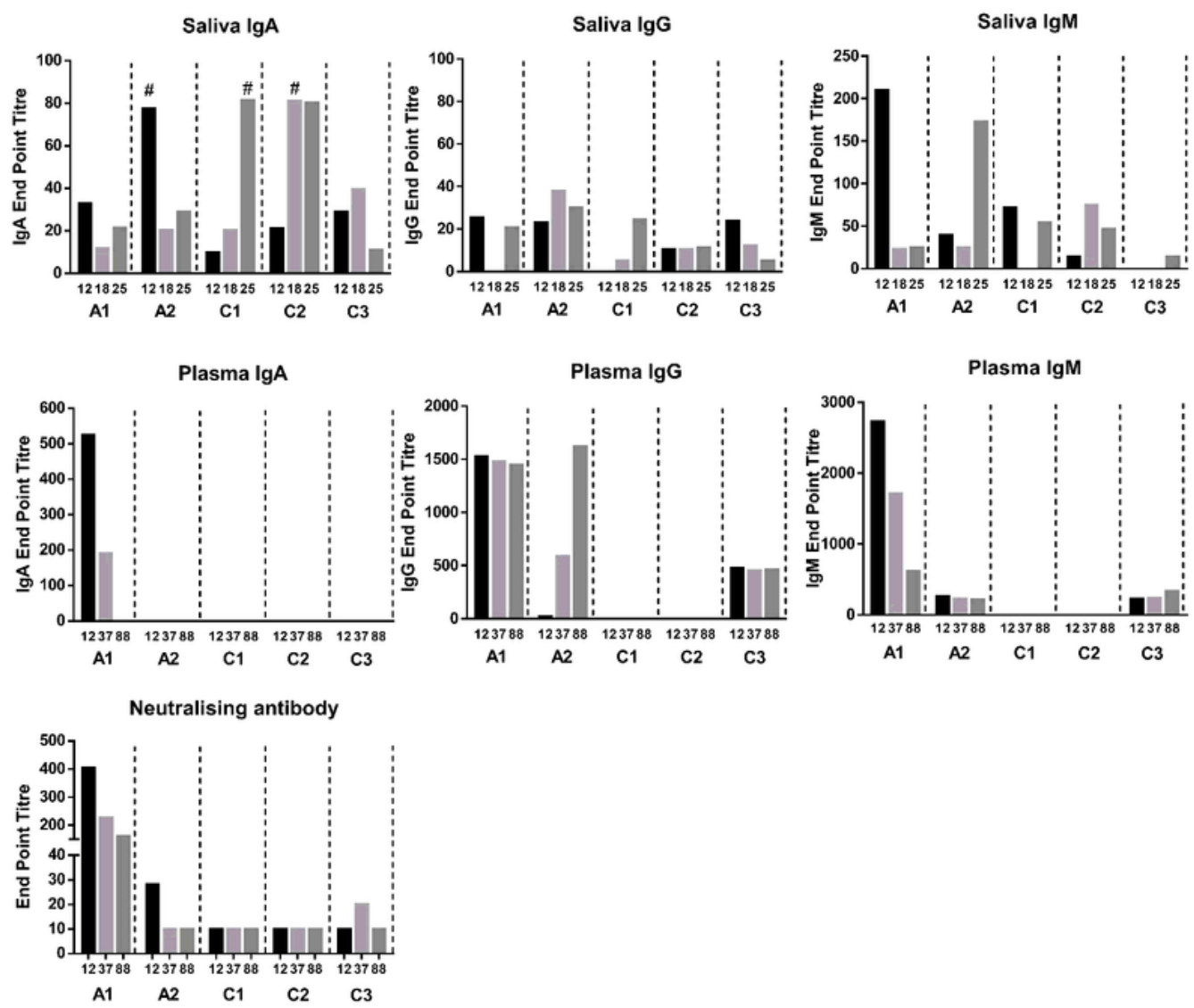

\section{Figure 3}

Saliva (day $12,18,25$ ) and plasma (day $12,37,88$ ) samples were analysed for antibody responses against SARS-CoV2 S1 protein by ELISA and by microneutralization assay. (A) Anti-S1 salivary IgA, IgG and IgM. \# IgA anti-S1 response that developed concurrent with resolution of symptoms. (B) Anti-S1 plasma IgA, IgG and IgM. (C) Neutralising antibody activity in plasma. A1: mother, A2: father, C1: male (9 years), C2: male (7 years), C3: female (5 years) 
A

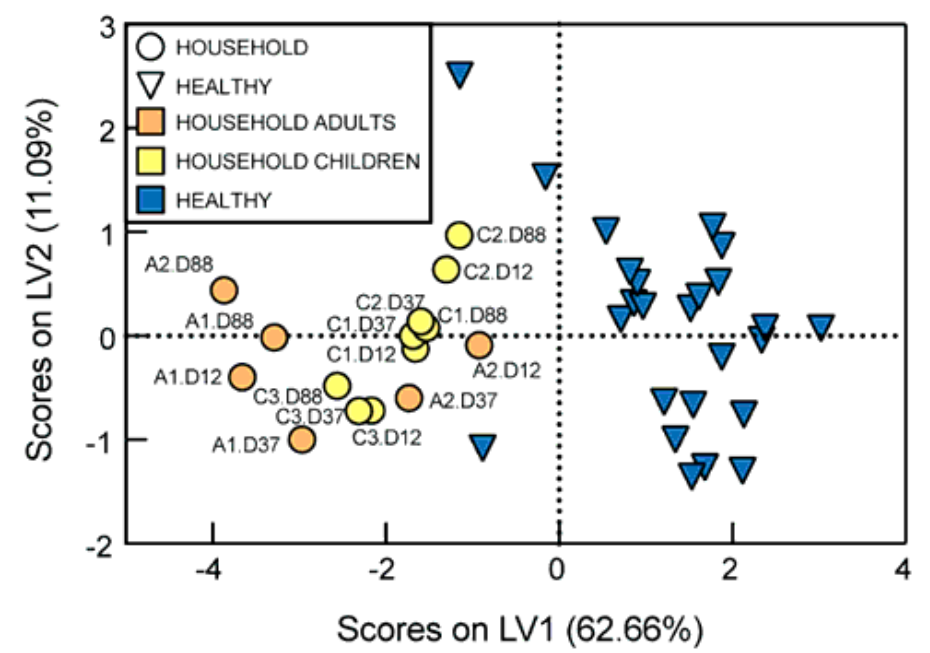

C

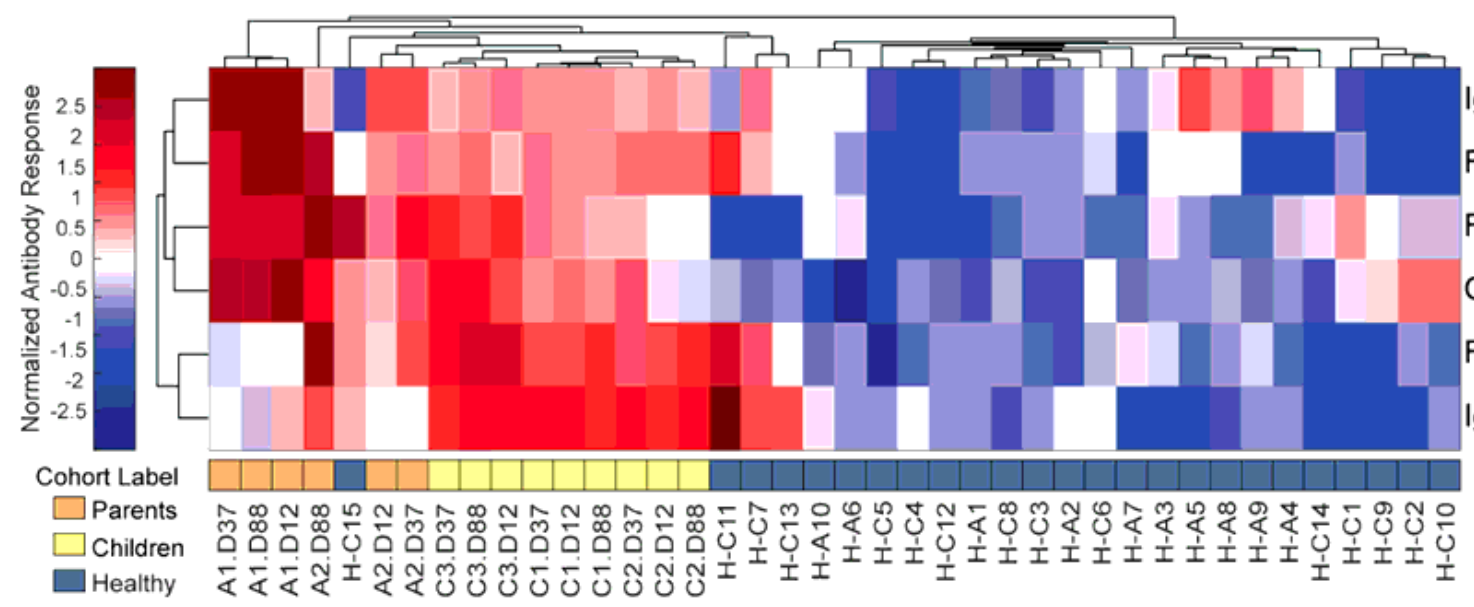

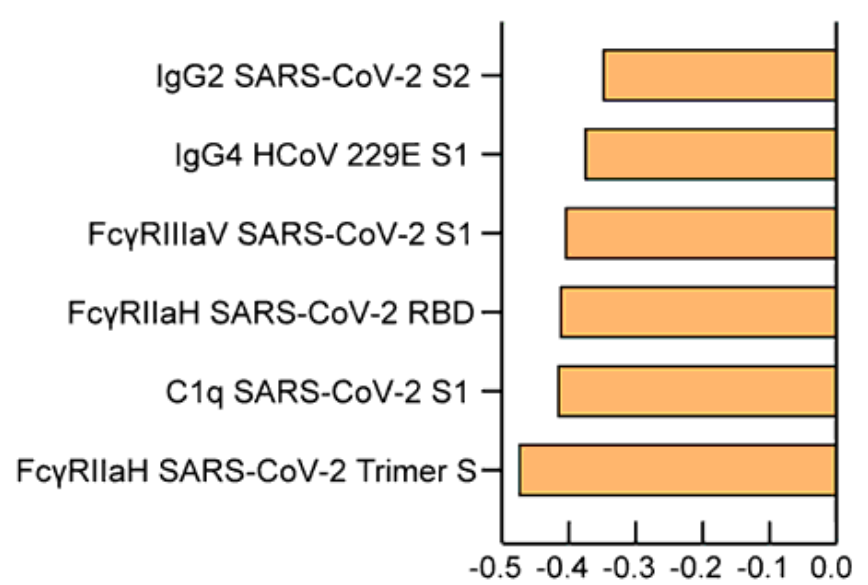

Loadings on LV1 $(62.66 \%)$

IgG4 HCoV 229E S1 FcyRllaH SARS-CoV-2 Trimer S FcyRIIlaV SARS-CoV-2 S1 C1q SARS-CoV-2 S1 FcYRIlaH SARS-CoV-2 RBD IgG2 SARS-CoV-2 S2

\section{Figure 4}

Family of symptomatic SARS-CoV-2 PCR positive parents and SARS-CoV-2 PCR negative children have distinct serological responses compared to healthy individuals, characterized by elevated SARS-CoV-2 specific responses. A. PLSDA scores plot of healthy (blue triangles) vs family (circles) containing both SARS-CoV-2 PCR positive parents (orange) and negative children (yellow) exhibited $98.0 \%$ calibration and $96.0 \%$ cross-validation accuracy, with $62.7 \%$ of variance explained by LV1 (x-axis). Family member samples are labelled with A (adult) or C (child) with day of sample collection listed after D. B. PLSDA plot of LV1 loadings driving the separation of groups, where negatively loaded features are associated with the family members. C. Hierarchical clustering of healthy individuals (blue) and family members (parents, orange; children, yellow) using a feature-selected serological signature, where red indicates a relatively high antibody response and blue a relatively low antibody response (z-score). Samples ( $\mathrm{x}$-axis) are labelled with $\mathrm{H}$ (healthy non-household members), and $\mathrm{A}$ (adult) or $\mathrm{C}$ (child). Day of sample collection is listed at the end of family member sample labels.

\section{Supplementary Files}


This is a list of supplementary files associated with this preprint. Click to download.

- LetterNatureMedExtendeddata.docx 